Ann. Zootech., I974, 23 (4), 537-554.

\title{
UTILISATION DE LA FÉVEROLE ENTIÈRE ET DÉCORTIQUÉE PAR LE PORG EN CROISSANCE : DIGESTIBILITÉ ET DISPONIBILITÉ DES ACIDES AMINÉS (1)
}

\author{
B. PASTUSZEWSKA, P. H. DUÉ**, Y. HENRY*, \\ D. BOURDON* et J. JUNG** \\ avec la collaboration technique de J. P. Hautducceur, Michèle Serezat \\ et Chantal Blondel \\ Polish Academy of Sciences, \\ Institute of Animal Phy'siology and Nutrition, \\ 05-110 Jablonna near Warsaw (Pologne) \\ * Station de Recherches sur l'Élevage des Porcs \\ ** Laboratoire de Physiologie de la Nutrition, \\ Centre national de Recherches zootechniques, I. N.R. A., \\ 78350 Jouy en Josas
}

\section{RÉSUMÉ}

Une étude est réalisée sur I 2 porcs mâles castrés Large White, d'un poids moyen de $25 \mathrm{~kg}$, en cages de métabolisme, dans le but de comparer, à celle du tourteau de soja 44 (lot I), 1'utilisation digestive et métabolique de la féverole de variété Pavane sous forme entière (lot 2) ou décortiquée (lot 3). Ces aliments constituent les seules sources azotées et sont incorporés dans des régimes semi-purifiés en remplacement de proportions variables d'amidon de maïs, afin d'assurer une teneur identique en matières azotées. Il est procédé, de plus, à une supplémentation des régimes en DL-méthionine, respectivement $0,05 \mathrm{p}$. Ioo et $0,15 \mathrm{p}$, Ioo dans le cas des régimes "soja " et "féverole".

La digestibilité apparente des composants de la ration est plus faible dans le lot 2 et, par rapport au lot $\mathbf{I}$, cette diminution est plus importante pour les nutriments azotés $(78,80$ contre $89,49)$ que pour les nutriments énergétiques $(84,49$ contre 89,79$)$.

Le décorticage de la graine de féverole améliore la digestibilité des différents nutriments (azote : 86, I7 ; énergie : 89,79). Par contre, l'utilisation métabolique de l'azote absorbé est plus faible dans le lot 3 (coefficient de rétention, p. Ioo : 51,26) que dans les deux autres lots $(64,73$ dans le lot I ; 60,04 dans le lot 2). En définitive, la quantité journalière d'azote retenu, qui est en relation étroite avec la vitesse de croissance des animaux, est plus élevée dans le lot I (I8, Io g) que dans les 2 lots renfermant la féverole (lot $2: 15,69 ; \operatorname{lot} 3: 13,68$ ),

Ces résultats sont confirmés, dans leur ensemble, à un stade plus tardif de la croissance $(65 \mathrm{~kg})$.

Les digestibilités apparentes et réelles des acides aminés des trois sources azotées ont été estimées et il s'avère que l'amplitude de leurs variations est plus élevée dans le lot 2 (féverole entière). Dans les 3 lots, il apparaît que les digestibilités apparentes ou réelles de l'arginine, de l'histidine, de l'acide glutamique sont plus élevées que celle de l'azote ; inversement, la thréonine, la valine, l'isoleucine, l'alanine et la glycine ont une digestibilité apparente ou réelle plus faible.

(1) Les demandes de tirages à part devront être envoyées à la Station de Recherches sur l'Élevage des Porcs. 
Enfin, la mesure des concentrations en aminoacides libres du sang fait apparaitre, dans le lot 3, une teneur plus élevée en lysine libre qui est en relation avec une quantité plus faible d'azote retenu.

\section{INTRODUCTION}

Des études récentes sur l'utilisation digestive de la féverole entière et décortiquée chez le Porc (Henry et Bourdon, I973) ou le Rat (VERMorel, I973 a et $b$ ), ont mis en évidence une amélioration sensible du coefficient d'utilisation digestive de l'énergie et de l'azote à la suite du décorticage de la graine. Cette influence favorable du décorticage peut être attribuée, soit à une diminution de la teneur en cellulose brute, localisée principalement dans les téguments, soit à une élimination des tannins moyennement polymérisés, présents en quantité importante dans ces téguments et pouvant avoir un effet dépressif sur l'absorption des nutriments (VERMOREL, I973 a).

Les travaux de ZEBROwska (I973), sur porcs munis de canules digestives ont montré que l'absorption des acides aminés au niveau de l'intestin grêle est plus faible dans le cas où les animaux reçoivent un régime renfermant de la féverole plutôt que du tourteau de soja. Dans le même temps, cet auteur observe une accumulation de matières azotées dans la dernière partie de l'intestin grêle, après un repas à base de féverole. On peut alors supposer qu'un des facteurs responsables de la valeur nutritionnelle plus faible de la féverole, en regard de celle du tourteau de soja, est la moins bonne disponibilité de certains acides aminés, qui peut être améliorée par le décorticage de la graine.

Dans le but de préciser la valeur nutritionnelle de la féverole décortiquée, une étude sur porcs en croissance a été entreprise, en comparant les utilisations digestive et métabolique de la féverole entière ou décortiquée à celles du tourteau de soja, ces matières azotées étant introduites comme seule source azotée dans des régimes de type semi-synthétique. L'estimation de la disponibilité des acides aminés repose d'une part, sur la détermination de leur digestibilité apparente ou réelle et d'autre part, sur l'étude des variations, suivant la source azotée, des teneurs en acides aminés libres du sang.

\section{MATÉRIEL, ET MÉTHODES}

\section{$1^{\text {re }}$ Expérience}

Douze porcs mâles castrés de race Large White, d'un poids moyen de $25 \mathrm{~kg}$, placés en cages de digestibilité, sont soumis pendant Io jours à un régime identique, de type semi-synthétique, à base de farine de hareng de Norvège. A l'issue de cette période préexpérimentale, ils sont répartis en 3 lots, suivant un dispositif en blocs et reçoivent leur régime propre. Après une semaine d'adaptation, les collectes de fèces et d'urine sont réalisées pendant ıo jours. Afin d'éviter leur fermentation, les fèces sont prélevées 3 fois par jour et conservées à une température d'environ $-{ }_{1} 5^{\circ} \mathrm{C}$; l'urine est collectée quotidiennement et conservée en milieu acide. A la fin de cette dernière période, un prélèvement de sang est effectué dans la veine cave antérieure, sur chaque animal, 5 heures après le premier repas en vue du dosage des acides aminés libres. A la suite de cette étude, 2 animaux par lot sont soumis à un régime protéiprive ; après une période d'adaptation de 4 jours, on procède, comme précédemment, à une collecte de fèces et d'urine, durant six jours, suivie d'un prélèvement de sang. 
Les caractéristiques des matières premières et la composition des régimes sont consignées dans les tableaux I et 2 . Les aliments étudiés, c'est-à-dire le tourteau de soja 44 (lot I), la féverole, de variété Pavane, sous forme entière (lot 2) ou décortiquée (lot 3), sont incorporés dans les régimes, en remplacement de proportions variables d'amidon de maïs, afin d'assurer une teneur identique en matières azotées. Compte tenu d'une déficience primaire des protéines de féverole et de soja en acides aminés soufrés, il est procédé à une supplémentation des régimes par la DL-méthionine, respectivement 0,05 p. Ioo et o, 5 p. Ioo clans le cas des régimes "soja " et "féverole". Les animaux des trois lots reçoivent les mêmes quantités des régimes expérimentaux, soit I, $3 \mathrm{~kg}$ par jour, distribués sous forme humide, trois fois par jour.

\section{TABLEAU I}

Composition chimique des matières premières

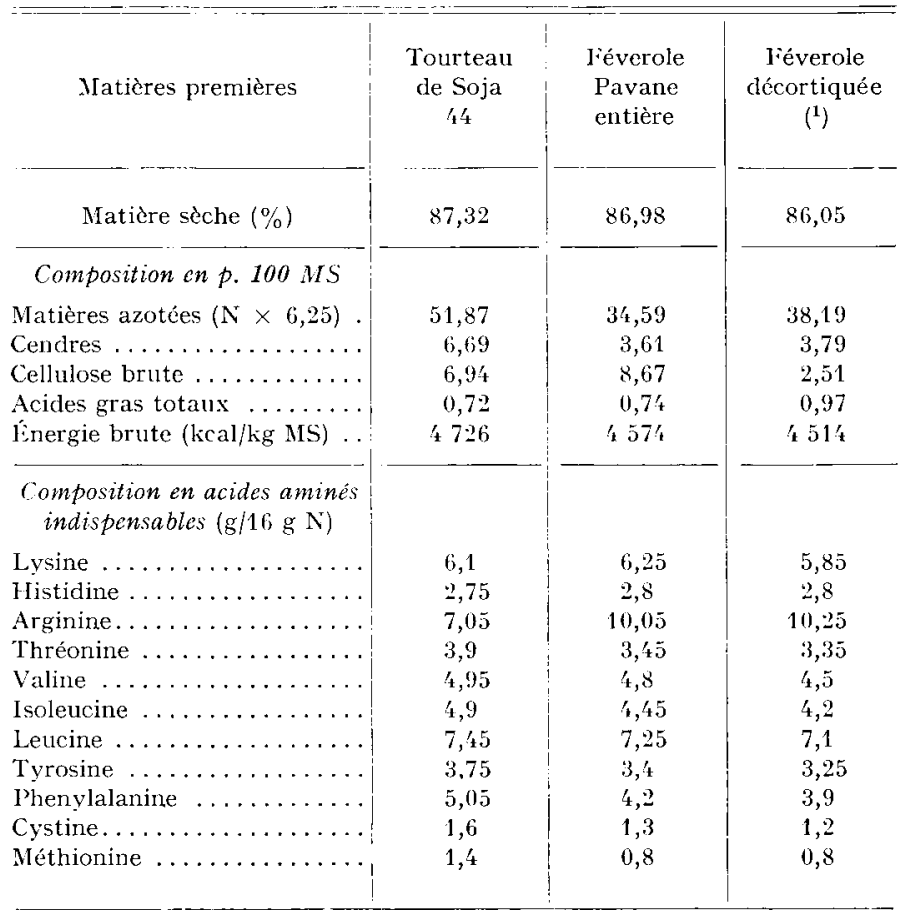

(1) Le pourcentage de téguments estimé sur un échantillon de graines est de 13,25 p. 100 dont la composition est la suivante :
Natière sèche : 88,95
Protéines $(\mathrm{N} \times 6,25): 4,73$
Cellulose brute : 58,42
Cendres
Énergie brute : ${ }_{4} 154 \mathrm{kcal} / \mathrm{kg}$ MS

Les teneurs en matière sèche, azote, cellulose brute, cendres et énergie des matières premières, régimes et fèces lyophilisécs sont estimées par des techniques déjà décrites (HENRY, RéRAT, I966), tandis que le closage des acides gras totaux dans les fèces est cffectué selon la méthode de Toult.EC et al., (I968). La composition en acides aminés des aliments (tabl. 3), des régimes et des fèces est déterminée par chromatographie sur colonne échangeuse d'ions, dans des conditions analytiques décrites par Pron et Fauconneau (1966); le tryptophane, détruit lors de l'hydrolyse acide, n'est pas dosé, tandis que les acides aminés soufrés sont oxydés, au préalable, par l'acide performique. L'extraction et le dosage des acides aminés libres du sang sont réalisés suivant une technique décrite par Duée et Rŕrat (I975). 
Dans tous les cas, le dosage des acides aminés est effectué sur des échantillons (fèces, aliments ou sang) individuels. Par contre, à la suite de l'étude avec le régime protéiprive, les mêmes dosages sont réalisés sur un échantillon moyen.

TABLEAU 2

Composition des régimes expérimentaux et résultats d'analyse

\begin{tabular}{|c|c|c|c|c|}
\hline Lot ou régime & $\begin{array}{c}1 \\
\text { Tourteau } \\
\text { de Soja }\end{array}$ & $\begin{array}{c}2 \\
\text { Féverole entière }\end{array}$ & $\begin{array}{c}3 \\
\text { Féverole } \\
\text { décortiquée }\end{array}$ & Protéiprive \\
\hline $\begin{array}{c}\text { Composition } \\
\text { (en p. } 100 \text { du régime) }\end{array}$ & & & & \\
\hline Tourteau de soja $44 \ldots \ldots \ldots \ldots \ldots$ & 33,5 & - & - & - \\
\hline Féverole entière $\ldots \ldots \ldots \ldots \ldots \ldots$ & $\ldots$ & 51 & - & - \\
\hline Féverole décortiquée $\ldots \ldots \ldots \ldots$ & - & - & 46 & 一 \\
\hline Amidon de maĩs . ............. & 49 & 31,5 & 36,5 & 83,5 \\
\hline Huile de mais $\ldots \ldots \ldots \ldots \ldots \ldots$ & 3 & 3 & 3 & 3 \\
\hline Cellulose de bois "Colmacel $\mathrm{F}_{2}$ " & 4 & 4 & 4 & 4 \\
\hline Sucre cristallisé $\ldots \ldots \ldots \ldots \ldots$ & 5 & 5 & 5 & 5 \\
\hline Mélange minéral $\left({ }^{1}\right) \ldots \ldots \ldots \ldots$ & 3,5 & 3,5 & 3,5 & 3,5 \\
\hline Mélange vitaminique $\left({ }^{2}\right) \ldots \ldots$ & 1 & 1 & 1 & 1 \\
\hline Prémélange "Méthionine $"\left({ }^{3}\right) \ldots$ & 1 & 1 & 1 & - \\
\hline \multicolumn{5}{|l|}{$\begin{array}{l}\text { Résultats analytiques } \\
\text { (en p. } 100 \mathrm{MS} \text { ) }\end{array}$} \\
\hline Matières sèches $(\%) \ldots \ldots \ldots$ & 90,06 & 88,07 & 88,08 & 88,87 \\
\hline Matières azotées $\ldots \ldots \ldots \ldots \ldots \ldots$ & 18,06 & 18,43 & 16,81 & 0,08 \\
\hline Cendres $\ldots \ldots \ldots \ldots \ldots \ldots \ldots \ldots$ & 5,77 & 5,47 & 5,14 & 3,76 \\
\hline Cellulose brute $\ldots \ldots \ldots \ldots$ & 6,29 & 8,26 & 5,24 & 4,50 \\
\hline Acides gras totaux $\ldots \ldots \ldots \ldots \ldots$ & 2,88 & 2,74 & 3,04 & 2,42 \\
\hline Énergie brute (kcal/kg MS) .... & 4287 & 4418 & $435 t^{\prime}$ & 4166 \\
\hline
\end{tabular}

(1) Composition du mélange minéral (p. 100 du régime) : phosphate bicalcique : 1,9; craie broyée : 0,7 ; sel marin : 0,25 ; sulfate de magnésie : 0,15 ; chlorure de potassium : 0,4 ; oligoéléments : 0,1 .

Composition du prémélange " oligoéléments " (g pour $1 \mathrm{~kg}$ de prémélange) : sulfate de fer, $7 \mathrm{H}_{2} \mathrm{O}: 400$; sulfate de manganèse, $\mathrm{H}_{2} \mathrm{O}$; 160 ; sulfate de cuivre, $5 \mathrm{H}_{2} \mathrm{O}: 40$; sulfate de zinc : 150 ; iodure de potassium : 0,3 ; craie broyée, qs p.

$\left.{ }^{2}\right)$ Composition du mélange vitaminique : quantités pour $1 \mathrm{~kg}$ de prémélange. Produit pur, introduit à raison de 1 p. 100 de la ration (en $\mathrm{mg}$ ) : thiamine : 220 ; riboflavine : 620 ; niacine : 2200 ; acide pantothénique : 2000 ; pyridoxine $\mathrm{HCl}: 260$; acide folique : 160 ; inositol : 40000 ; acide paraminobenzoïque : 4400 ; biotine : 12 ; vitamine B 12 (à $100 \mathrm{mg} / \mathrm{kg}: 2,2$; choline (à 25 p. 100) : 200000 ; vitamine $\mathrm{K}: 440$; tocophérols : 16000 ; vitamine A $(50000 \mathrm{UI} / \mathrm{g}): 500000 \mathrm{UI}$; vitamine $\mathrm{D}_{\mathrm{a}}(100000 \mathrm{UI} / \mathrm{g}): 100000 \mathrm{UI}$; amidon, qsp.

(3) Prémélange " Méthionine ", à base d'amidon de maïs, renfermant 0,05 p. 100 de DL-méthionine (lot 1) ou 0,15 p. 100 (lots 2 et 3 ).

\section{2e Expérience}

Un deuxième essai est réalisé à un stade plus tardif de la croissance $(65 \mathrm{~kg})$, sur 9 animaux, suivant le même schéma expérimental. Les trois régimes utilisés ont les mêmes caractéristiques que dans la première expérience (tabl. 2) et sont distribués à raison de $\mathrm{I}, 9 \mathrm{~kg}$ par jour. Après une semaine d'adaptation, les collectes de fèces et d'urine sont réalisées pendant ıo jours. Seules les observations portant sur l'utilisation digestive et métabolique des nutriments, autres que les acides aminés, sont renouvelées. 


\section{RÉSULTATS}

Les résultats de composition en acides aminés indispensables de la féverole (tabl. I) ne font apparaître aucune différence liée au décorticage, ce qui était prévisible, compte tenu du faible taux azoté dans les téguments. Par rapport au tourteau de soja, les teneurs sont légèrement plus faibles pour la thréonine, l'isoleucine, la phénylalanine et les acides aminés soufrés, ce qui est en accord avec les données de Pion et FAucoriseau (Ig66), tandis que l'arginine se trouve à un taux plus élevé. Les mêmes différences s'observent au niveau de la composition en acides aminés des régimes (tabl. 3) mais avec des teneurs légèrement plus faibles en valeur absolue.

TABIEAU 3

Composition en acides aminés des régimes expérimentaux

(en g p. $\mathbf{1} 6 \mathrm{~g} \mathrm{~N}$ )

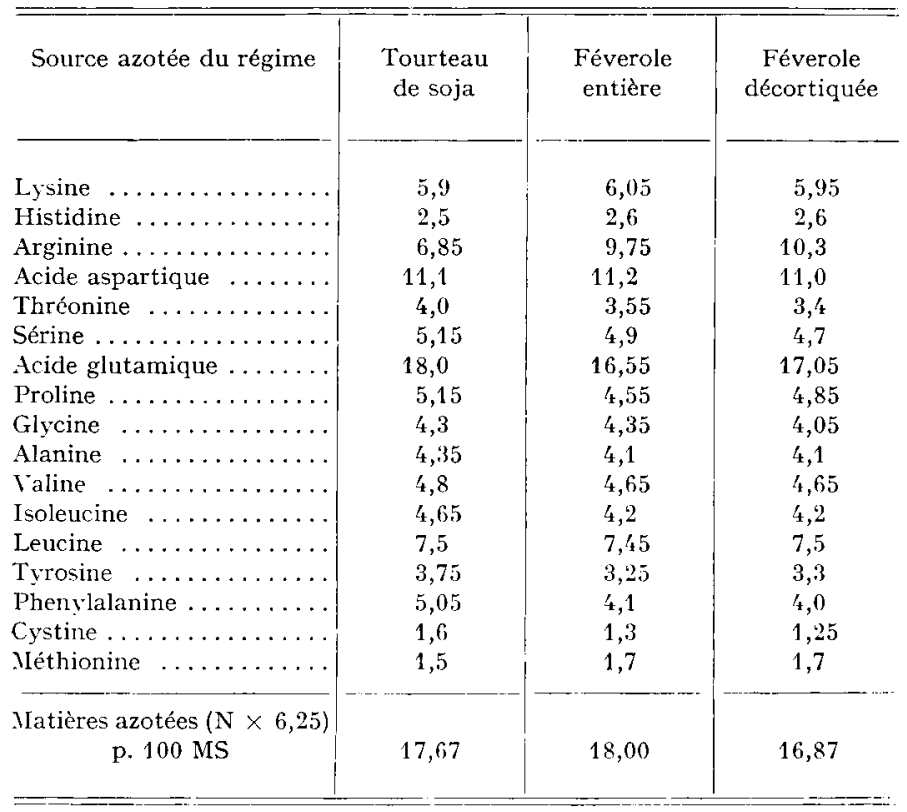

\section{I. - Utilisation digestive ct métabolique de l'azote et de l'énergie}

\section{a) Expérience 1.}

La comparaison des quantités journalières ingérées par les animaux, en nutriments énergétiques et azotés (tab1. 4), montre un léger écart entre les lots I et 2 d'une part, et le lot 3 d'autre part. Il apparaît, en effet, une diminution de la consommation des porcs du lot 3 ("féverole décortiquée ") d'environ 8 p. Ioo pour l'azote et 2 p. Ioo pour 1'énergie, par rapport à celle des animaux du lot I (" tourteau de soja »). 
La digestibilité apparente des composants de la ration est plus faible dans le lot 2 (" féverole entière ") et, par rapport au lot $\mathrm{I}$, cette diminution est plus importante pour les nutriments azotés (I0,7 points) que pour les nutriments énergétiques $(5,5$ points pour la matière sèche et pour la matière organique ; 5,3 points pour l'énergie). Par contre, aucune différence n'est enregistrée entre les lots, en ce qui concernela digestibilité des acides gras totaux (moyenne : 83,24).

\section{TABL,EAU 4}

Utilisation digestive et métabolique de l'azote et de l'énergie

(expérience I)

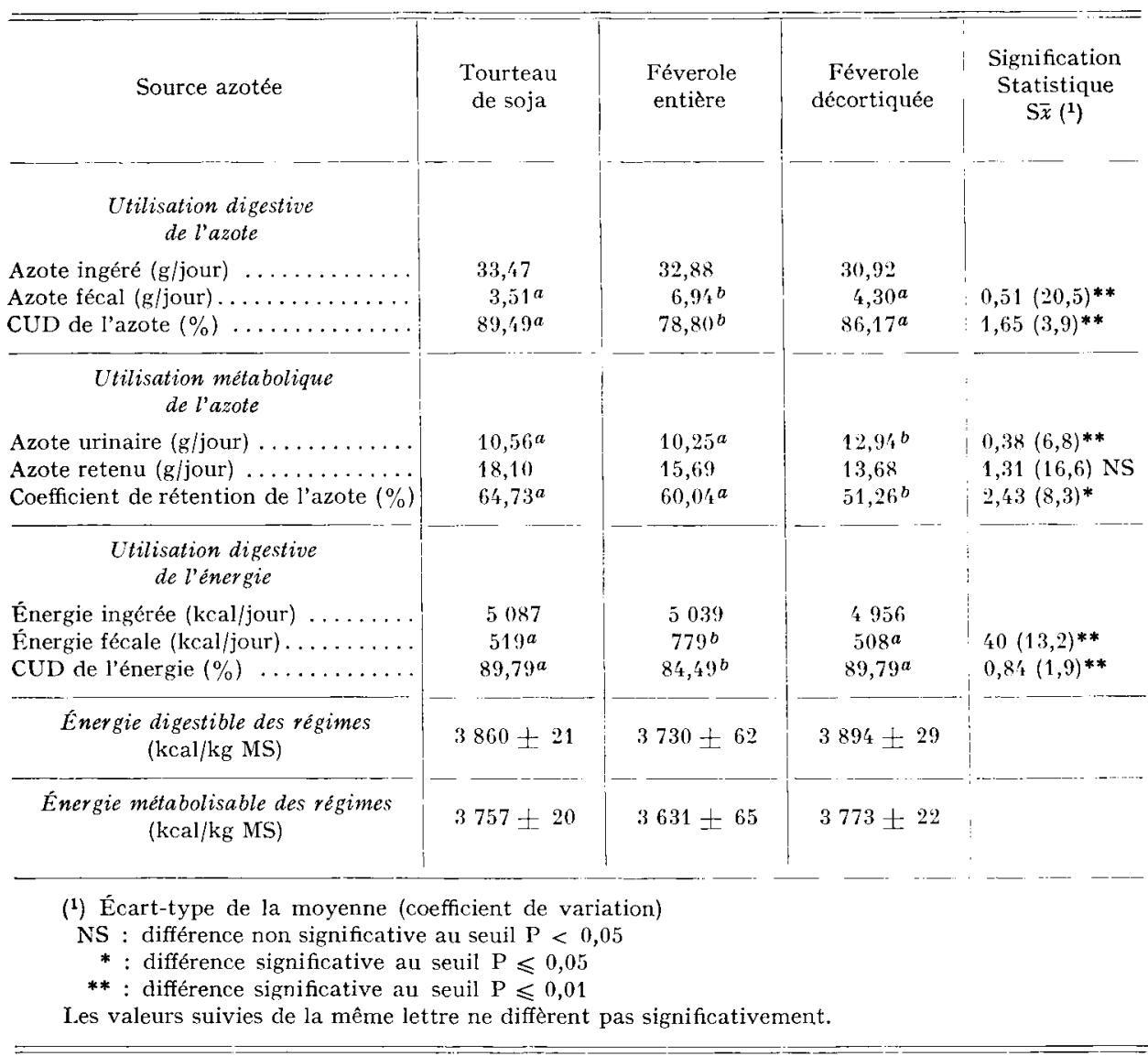

Le remplacement total dans le régime de la féverole entière par la féverole décortiquée améliore la digestibilité des différents nutriments : 1'utilisation digestive de l'énergie, dans le lot 3 (" féverole décortiquée ») est comparable à celle du lot I (" tourteau de soja ") ; la digestibilité des nutriments azotés, quoique non statistiquement différente, est légèrement plus faible en regard de celle du lot $I$.

I1 ressort, par conséquent, une amélioration de l'utilisation digestive des nutriments azotés et énergétiques après décorticage de la graine de féverole et cette diges- 
tibilité est comparable à celle du régime renfermant comme seule source azotée le tourteau de soja.

Les résultats présentés dans le tableau 4 mettent en évidence une excrétion d'azote dans l'urine, chez les animaux du lot 3 (" féverole décortiquée "), plus importante que chez ceux des deux autres lots. Il s'ensuit, dans ce lot, une diminution statistiquement significative de l'utilisation métabolique de l'azote absorbé, qui se répercute sur la quantité d'azote déposée quotidiennement dans l'organisme ; toutefoi 3 , dans ce dernier cas, les différences ne sont plus significatives au seuil 0,05 .

Rien de comparable n'est observé en ce qui concerne le pourcentage d'énergie mátabolisable par rapport à l'énergie digestible, dont les valeurs sont voisines dans les 3 lots, soit 97 p. roo. Ainsi, les valeurs énergétiques des régimes I (" tourteau de soja ") et 3 (" féverole décortiquée »), exprimées en énergie digestible ou en énergie mútabolisable, sont comparables entre elles et légèrement plus élevées que celles du régime renfermant la féverole entière (lot 2).

En définitive, l'utilisation de la féverole entière ou décortiquée en remplacement total du tourteau de soja dans le régime de porcs en croissance entraine un abaissement de 1'utilisation pratique de 1'azote ( $\left.\frac{\text { azote retenu }}{\text { azote ingéré }}\right)$, par diminution soit, de la digestibilité apparente (lot 2 : féverole entière), soit du coefficient de rétention (lot 3 : féverole décortiquée) des matières azotées. Ceci est à rapprocher des différences obtenues dans la vitesse de croissance des animaux, estimée durant la période expérimentale de Io jours : le gain moyen quotidien des animaux du lot I est de $620 \pm 6 \mathrm{I} \mathrm{g}$, significativement supérieur à celui des animaux du lot $2(485 \pm 36 \mathrm{~g})$ ou du lot $3\left(43^{\circ} \pm 44 \mathrm{~g}\right)$.

A partir des valeurs en énergie digestible et métabolisable des différents régimes et du régime protéiprive, il est possible de calculer les valeurs énergétiques des sources azotées utilisées dans cette expárience, c'est-à-dire du tourteau de soja, de la féverole entière et décortiquée.

Compte tenu de la valeur en énergie digestible du régime protéiprive $(35 \mathrm{r} 3 \mathrm{kcal} /$ $\mathrm{kg})$, un système de 3 équations à 3 inconnues $(x, y, z$, étant respectivement les valeurs en énergie digestible du tourteau de soja, de la féverole entière, de la féverole décortiquée, en $\mathrm{kcal} / \mathrm{kg})$ et à un seul paramètre $(a$, valeur en énergie digestible de l'amidon de maïs) est ainsi établi :

$$
\left\{\begin{array}{l}
x=a+3 \\
y=a-26 \text { I } \\
z=a+\text { I3 }
\end{array}\right.
$$

Si l'on se base sur les résultats de Schizuann, Hoffmann et Nehring (Ig6r), la valeur en énergie digestible de l'amidon de maïs peut être estimée à $4000 \mathrm{kcal} / \mathrm{kg}$ de matière sèche, correspondant à $3500 \mathrm{kcal} / \mathrm{kg}$ d'aliment frais. De ce fait, les valeurs en énergie digestible du tourteau de soja, de la féverole entière et décortiquée sont respectivement de $3503,3239,35^{1} 3 \mathrm{kcal} / \mathrm{kg}$, soit $4000,3700,4040 \mathrm{kcal} / \mathrm{kg} \mathrm{MS}$.

De la même manière, d'après les valeurs en énergie métabolisable du régime protéiprive $\left(3477 \mathrm{kcal} / \mathrm{kg}\right.$ ) et de 1'amidon de mais $\left(345^{\circ} \mathrm{kcal} / \mathrm{kg}\right)$, les estimations des valeurs en énergie métabolisable des trois sources azotées sont respectivement de $3283,3083,3304 \mathrm{kcal} / \mathrm{kg}$ ou $3750,3530,3800 \mathrm{kcal} / \mathrm{kg}$ MS. 
b) Expérience 2 .

Les résultats de cette étude, portant sur 3 animaux par lot, sont consignés dans le tableau 5 . Comme précédemment, la digestibilité apparente des composants de la ration est plus faible dans le lot 2 ("féverole entière ") et, par rapport au lot I (" tourteau de soja $n$, cette diminution est plus importante pour les nutriments azotés (5,3 points) que pour les nutriments énergétiques (3,6 points). Toutefois, la différence entre les deux lots est plus faible à ce stade qu'au stade précédent, ce qui semble indiquer, en comparaison avec le lot témoin, une meilleure utilisation digestive de la féverole entière à $65 \mathrm{~kg}$ plutôt qu'à $30 \mathrm{~kg}$. Le remplacement total de la féverole entière par la féverole décortiquée améliore la digestibilité des différents nutriments qui dépasse même les digestibilités correspondantes du lot I. L'excrétion azotée urinaire est significativement plus faible dans le lot 2 (" féverole entière ") et, quoique les quantités journalières d'azote retenu ne soient pas significativement différentes suivant les lots, le coefficient de rétention de l'azote est amélioré avec le régime renfermant la féverole entière, ce qui n'avait pas été montré au premier stade de l'étude.

TABLEAU 5

Utilisation digestive et métabolique de l'azote et de l'énergie (expérience 2)

\begin{tabular}{|c|c|c|c|c|}
\hline Source azotée & $\begin{array}{l}\text { Tourteau } \\
\text { de soja }\end{array}$ & $\begin{array}{l}\text { Péverole } \\
\text { entière }\end{array}$ & $\begin{array}{l}\text { léverole } \\
\text { décortiquée }\end{array}$ & $\begin{array}{c}\text { Signification } \\
\text { statistique } \\
S \bar{x}\left({ }^{1}\right)\end{array}$ \\
\hline $\begin{array}{l}\text { Utilisation digestive } \\
\text { de l'azote }\end{array}$ & & & & 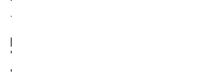 \\
\hline Azote ingéré (g/jour) & 48,87 & 48,20 & $4,6,19$ & $\vdots$ \\
\hline Azote fécal (g/jour) & $5,58^{a}$ & $8,07^{b}$ & $4,42^{a}$ & $0,49(1,4)^{*}$ \\
\hline CUD de l'azote $(\%) \ldots \ldots$ & $88,58 a$ & $83,2,1^{b}$ & $90,4^{1} 8^{a}$ & $1,13(2,2)^{*}$ \\
\hline \multicolumn{5}{|l|}{$\begin{array}{l}\text { Utilisation métabolique } \\
\text { de l'azote }\end{array}$} \\
\hline Azote urinaire (g/jour) $\ldots \ldots$ & $21,02^{a}$ & $15,61^{b}$ & $22,42 a$ & $10,97(8,5)^{*}$ \\
\hline Azote retenu (g/jour) $\ldots \ldots \ldots \ldots \ldots$ & 22,27 & 24,52 & 19,65 & $0,94(7,3) \mathrm{NS}$ \\
\hline Coefficient de rétention de l'azote $(\%)$ & $51,42^{b}$ & $61,2 \geq a$ & $46,64^{b}$ & $2,11(1 ;, 8)^{*}$ \\
\hline \multicolumn{5}{|l|}{$\begin{array}{l}\text { Utilisation digestive } \\
\text { de l'énergie }\end{array}$} \\
\hline Énergie ingérée $(\mathrm{kcal} / \mathrm{jour}) \ldots \ldots \ldots$ & 7427 & 7326 & 7451 & ! \\
\hline Énergie fécale $(\mathrm{kcal} /$ jour $) \ldots \ldots \ldots$ & $687^{a b}$ & $972^{a}$ & $538^{b}$ & $76(17,9)^{*}$ \\
\hline CUD de l'énergie $(\%) \ldots \ldots \ldots \ldots$ & $90,35^{a b}$ & $86,76^{b}$ & $92,77^{a}$ & $1,06(2,05)^{*}$ \\
\hline $\begin{array}{c}\text { Énergie digestible des régimes } \\
(\mathrm{kcal} / \mathrm{kg} \mathrm{MS})\end{array}$ & $3900 \pm 28$ & $3796 \pm 72$ & $4023 \pm 57$ & \\
\hline $\begin{array}{l}\text { Energie métabolisable des régimes } \\
\text { (kcal/kg MS) }\end{array}$ & $3778+19$ & $369 r_{4} \pm 67$ & $3895 \pm$ & \\
\hline
\end{tabular}

(1) Voir tableau 4. 
D'autre part, comme à $30 \mathrm{~kg}$ de poids vif, l'utilisation métabolique de l'azote dans le lot 3 ("féverole décortiquée ") est plus faible par rapport au lot I. Enfin, en ce qui concerne le pourcentage d'énergie métabolisable par rapport à l'énergie digestible, les valeurs obtenues ne sont pas différentes suivant les lots et voisines de $97 \mathrm{p}$. Ioo.

En résumé, il semble qu'à ce stade de la croissance ( $65 \mathrm{~kg}$ de poids vif), l'utilisation digestive et métabolique de la féverole entière soit améliorée. Le remplacement total dans le régime de la féverole entière par la féverole décortiquée améliore la digestibilité des nutriments mais semble déprécier l'utilisation métabolique de l'azote dans le cas considéré.

\section{2. - Digestibilités apparente et réelle des acides aminés}

Les digestibilités apparentes des acides aminés des différentes sources azotées ont été calculées, à partir des analyses en aminoacides des régimes d'une part, et des fèces récoltées pendant ro jours et sur 3 animaux par lot d'autre part (tabl. 6). D'une manière générale, les digestibilités apparentes de chaque acide aminé, pour chaque source azotée, peuvent être comparées à la valeur moyenne représentée par la digestibilité apparente de l'azote. I1 s'avère que l'amplitude de leurs variations est la même si 1'on considère le groupe des acides aminés indispensables et celui des acides aminés non indispensables.

En ce qui concerne les acides aminés indispensables, il apparaît, d'abord, que les digestibilités apparentes de l'arginine et, à un moindre degré, de l'histidine sont plus élevées, dans chaque lot, que les valeurs obtenues pour l'azote : l'écart observé entre les digestibilités apparentes de l'arginine et de l'azote varie de 4,4 à I I ,6 points suivant le régime. Certains autres acides aminés indispensables ont, d'autre part, une digestibilité apparente plus faible que la valeur moyenne obtenue pour l'azote. C'est le cas de la thréonine, de la valine, de l'isoleucine dans les trois lots et de la cystine dans les deux lots à base de féverole. Enfin, pour les autres acides aminés indispensables, la lysine en particulier, les valeurs enregistrées sont voisines de la digestibilité apparente de l'azote.

Dans le groupe des acides aminés non indispensables, l'alanine et, à un moindre degré, la glycine ont une digestibilité apparente plus faible que la valeur moyenne obtenue pour l'azote : l'écart des digestibilités, pour l'alanine, varie de 3,9 à 8 , I points suivant le lot considéré.

Les autres acides aminés non indispensables ont une digestibilité apparente at moins égale à celle de l'azote ; l'acide glutamique ayant, dans ce groupe, la digestibilité apparente la plus élevée.

La dispersion des digestibilités apparentes autour de celle de 1'azote total est plus élevée dans le lot 2 ò̀ le régime contient de la féverole entière. Il ne semble pas y avoir d'interaction entre la source azotée utilisée et la digestibilité apparente de chaque acide aminé par rapport à celle de l'azote total sauf, dans le cas présent, pour la méthionine dont l'utilisation digestive, par rapport aux autres acides aminés indispensables, paraît être améliorée dans le cas des régimes à base de féverole (lots 2 et 3 ). Ceci peut être une conséquence d'un apport plus important de DL-méthionine, sous forme libre, dans les régimes 2 et 3 que dans celui renfermant le tourteau de soja (tabl. 2). 
B. PASTUSZEWSKA ET COLL.

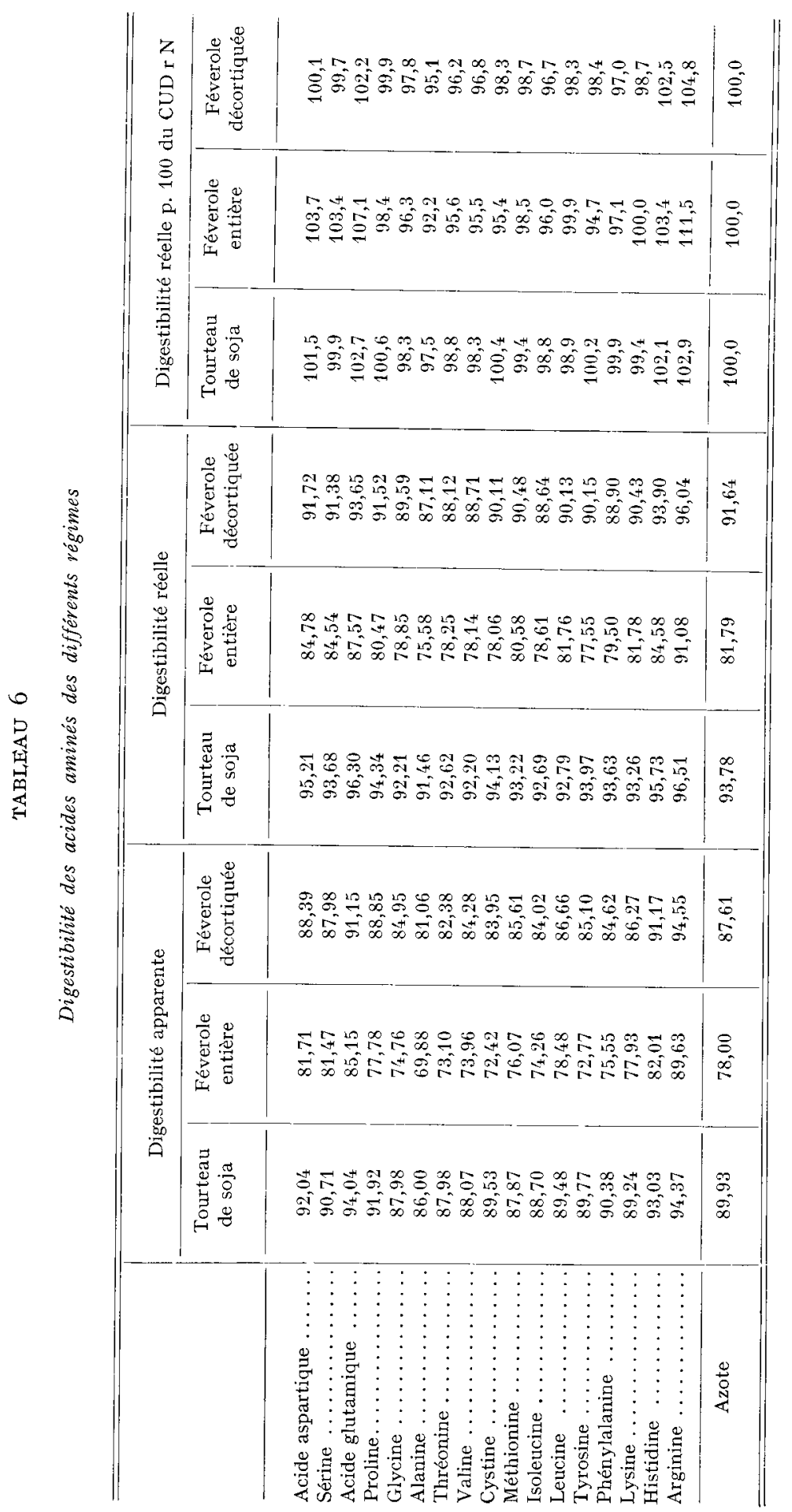


Les teneurs en acides aminés des fèces excrétées pendant la période où les animaux recevaient le régime protéiprive peuvent constituer une approche dans la détermination de la composition en acides aminés de l'azote endogène. Ces teneurs ont été exprimées en pourcentage de l'azote ou pour Ioo grammes de matière sèche ingérée (tableau 7), mais il est à noter que, le régime protéiprive renfermant $0,078 \mathrm{p}$. 100 de matières azotées par rapport à la matière sèche, les valeurs obtenues peuvent entraîner une légère surestimation de la dépense endogène en acides aminés.

\section{TABLEAU 7}

Excrétion endogène des acides aminés

\begin{tabular}{|c|c|c|}
\hline & $\begin{array}{c}\text { Teneur en acides } \\
\text { aminés des fèces } \\
\text { du lot " protéiprive " } \\
\text { (g/16 g N })\end{array}$ & $\begin{array}{c}\text { Excrétion endogène } \\
\text { d'acides aminés } \\
\text { (mg/100 g MS ingérée) }\end{array}$ \\
\hline Acide aspartique $\ldots \ldots \ldots$ & 9,2 & 62 \\
\hline Sérine $\ldots \ldots \ldots \ldots \ldots$ & $t_{1}, 0$ & 27 \\
\hline Acide Glutamique. . . . . . . & 10,5 & 72 \\
\hline Proline $\ldots \ldots \ldots \ldots \ldots \ldots$ & 3,3 & 22 \\
\hline Glycine $\ldots \ldots \ldots \ldots \ldots$ & 4,7 & 32 \\
\hline Alanine $\ldots \ldots \ldots \ldots \ldots$. & 6,2 & 42 \\
\hline Thréonine............. & 4,8 & 33 \\
\hline Valine $\ldots \ldots \ldots \ldots \ldots$ & 5,2 & 35 \\
\hline Cystine $\ldots \ldots \ldots \ldots \ldots$ & 2,0 & 13 \\
\hline Méthionine $\ldots \ldots \ldots \ldots$ & 2,1 & 14 \\
\hline Isoleucine $\ldots \ldots \ldots \ldots$ & 4,8 & 33 \\
\hline Leucine............ & 6,5 & 44 \\
\hline Tyrosine $\ldots \ldots \ldots \ldots \ldots$ & 4,1 & 28 \\
\hline Phenylalanine $\ldots \ldots \ldots \ldots$ & 4,3 & 29 \\
\hline 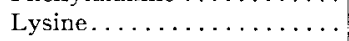 & 6,2 & 42 \\
\hline Histidine $\ldots \ldots \ldots \ldots \ldots$ & 1,7 & 12 \\
\hline Arginine $\ldots \ldots \ldots \ldots \ldots$ & 3,8 & 26 \\
\hline Azote & & 109 \\
\hline
\end{tabular}

Cette analyse permet, néanmoins, le calcul des digestibilités réelles des matières azotées et des différents acides aminés; une présentation des écarts entre les digestibilités des différents acides aminés et celle de l'azote, en pourcentage de la digestibilité réelle de l'azote, a, d'autre part, été réalisée (tab1. 6). Les conclusions tirées antérieurement, tant en ce qui concerne les acides aminés indispensables que les acides aminés non indispensables, sont maintenues quand on considère non plus la digestibilité apparente mais la digestibilité réelle des acides aminés. Il apparaît que la présence de la féverole entière dans le régime accroît la dispersion des digestibilités réelles des différents acides aminés, par rapport à la valeur obtenue pour l'azote. De la même façon, les digestibilités réelles de l'arginine, de l'histidine et de l'acide glutamique sont plus élevées que celles des matières azotées, contrairement à l'alanine. 


\section{3. - Variations des concentrations en acides aminés libres du sang suivant la source azotée du régime}

Les teneurs en acides aminés libres du sang sont rapportées dans le tableau 8. Il n'a pas été fait mention des résultats concernant le niveau de cystine, la teneur en cet acide aminé étant très faible et pratiquement non déterminable.

\section{TABLEAU 8}

Concentrations en acides aninés libres du sang

(en $\mathrm{mg} / \mathrm{I}$ oo $\mathrm{g}$ de sang frais)

\begin{tabular}{|c|c|c|c|c|}
\hline Régime & $\begin{array}{c}\text { Tourteau } \\
\text { de Soja }\end{array}$ & $\begin{array}{l}\text { Féverole } \\
\text { entière }\end{array}$ & $\begin{array}{c}\text { Féverole } \\
\text { décortiquée }\end{array}$ & Protéiprive \\
\hline Acide aspartique & 0,89 & 0,86 & 0,91 & 0,59 \\
\hline Sérine $\ldots \ldots \ldots$ & 2,18 & 2,27 & 2,94 & 1,79 \\
\hline Acide glutamique & 2,48 & 2,17 & 1,96 & 0,98 \\
\hline Proline $\ldots \ldots \ldots$ & 3,75 & 3,69 & 3,77 & 2,63 \\
\hline Glycine........ & 7,78 & 8.31 & 9,07 & 7,98 \\
\hline Alanine..... & 4,79 & '́,83 & 5,95 & 5,02 \\
\hline Thréonine ...... & 2,17 & 2,68 & 2,46 & 0,70 \\
\hline Valine......... & 3,72 & 3,29 & 3,39 & 1,43 \\
\hline Méthionine ..... & 0,62 & 0,89 & 0,85 & 0,50 \\
\hline Isoleucine $\ldots \ldots$ & 2,26 & 1,98 & 1,90 & 0,72 \\
\hline Leucine . . . . . . . & 2,74 & 2,71 & 2,69 & 1,33 \\
\hline Tyrosine........ & 2,06 & 1,93 & 2,16 & 0,88 \\
\hline Phenylalanine ... & 1,71 & 1,23 & 1,68 & 0,87 \\
\hline Lysine $\ldots \ldots \ldots$ & 4,00 & 3,99 & 4,91 & 3,21 \\
\hline Histidine . . . . . & $1,1,8$ & 1,86 & 2,35 & 1,17 \\
\hline Arginine $\ldots .$. & 1,81 & 2,36 & 2,24 & 0,48 \\
\hline $\begin{array}{c}\text { Somme des acides } \\
\text { aminés } \\
\text { indispensables }\end{array}$ & 22,57 & 22,92 & 24,63 & 11,29 \\
\hline
\end{tabular}

La somme des concentrations en acides aminés libres dans le sang apparaît plus élevée dans le lot 3 (féverole décortiquée). Cette différence n'est pas imputable aux concentrations en acides aminés non indispensables qui, en effet, ne semblent pas affectées par la nature de la source azotée du régime. Il est vrai que d'importants remaniements interviennent dans l'organisme, après l'absorption, et qui touchent en premier lieu les acides aminés non indispensables.

Par contre, l'examen des concentrations en acides aminés indispensables, dans le sang systémique, 5 heures après le repas dans le cas présent, met en évidence un accroissement de la teneur en lysine libre du sang dans le lot 3. De même, les teneurs en histidine et en méthionine sont plus élevées dans les lots 2 et 3 où le régime est à base de féverole décortiquée ou non.

En ce qui concerne les concentrations sanguines en acides aminés libres des animaux recevant le régime protéiprive, on peut constater une diminution de l'ensemble des teneurs de ces acides aminés, indispensables ou non, sauf pour la glycine, l'alanine, la lysine et, à un moindre degré, la méthionine. 


\section{DISCUSSION}

\section{I. -- Utilisation digestive comparée de la féverole entière et du tourteau de soja; influence du décorticage}

Les résultats de la présente étude, en ce qui concerne la comparaison de l'utilisation digestive de la féverole entière et du tourteau de soja, font ressortir une diminution importante de la digestibilité des nutriments (azotés, énergétiques) quand le régime renferme la féverole comme seule source azotée. Cette dépression de la digestibilité est plus marquée que dans des essais précédents (HENRY et BouRdon, I973) et affecte surtout les nutriments azotés. Il est vrai que, dans le cas présent, le taux d'introduction dans la ration de la féverole entière est beaucoup plus élevé (5I p. roo) que dans l'expérience citée précédemment.

Ceci est en accord avec les résultats obtenus sur le Rat (VERMOREL, I973 a) mettant en évidence une baisse plus importante de la digestibilité des nutriments azotés (I2,3 points) que des nutriments énergétiques ( 4,8 points). Quoi qu'il en soit, l'utilisation digestive de la féverole crue chez le Porc est supérieure à celle observée chez le Rat.

Le remplacement de la féverole entière par la féverole décortiquée a pour effet d'améliorer l'utilisation digestive des nutriments, ce qui avait déjà été montré par ailleurs (HENRY et BOURDON, I973). Le décorticage de la graine de féverole permet une augmentation de l'utilisation digestive des matières azotées, qui devient comparable à celle du tourteau de soja. On peut penser que l'effet dépressif des téguments sur le coefficient d'utilisation digestive de l'azote (CUDN) est dî̀, en grande partie, à leur teneur élevée en tannins, qui sont pratiquement absents dans les cotylédons (MARTIN, communication personnelle), ou à la présence d'autres facteurs (antitrypsiques par exemple).

Parallèlement, on peut associer l'augmentation de 5,3 points du CUD de l'énergie à la diminution de 3 points du taux de cellulose brute dans la ration, soit un accroissement de $I, 7$ point du CUD de l'énergie par point de cellulose, soit légèrement plus que la valeur de I,2 déterminée précédemment (HrNRy et Bournor, I973). Toutefois, la diminution du taux de cellulose brute dans la ration renfermant la féverole décortiquée n'explique pas, à elle seule, l'amélioration del'utilisation digestive de l'énergie puisque, pour un même CUD de l'énergie le régime I (tourteau de soja) a une teneur en cellulose brute supérieure de $\mathbf{I}$ point à celle du régime 3 (féverole décortiquée).

Les résultats de la seconde expérience, menée sur des animaux plus lourds $(65 \mathrm{~kg})$, confirment les conclusions tirées précédemment. Cependant, si la digestibilité du tourteau de soja ne semble pas modifiée entre les 2 stades de l'étude, il n'en est pas de même de celle de la féverole entière ou décortiquée, qui sont anéliorées au stade le plus tardif de la croissance.

Il en résulte que l'écart de digestibilité entre le tourteau de soja et la féverole entière diminue avec l'âge, ce qui semble traduire une meilleure utilisation de cette source azotée chez des animaux plus vieux comme le confirment d'ailleurs les essais d'alimentation en lot (HENRY et BouRdon, I972). Il est à remarquer que le mode 
d'estimation de la valeur énergétique des trois sources azotées, dans la première expérience, pourrait souffrir d'une certaine imprécision, compte tenu des modifications du taux d'humidité des composants, résultant de la non contemporanéité des périodes de collectes effectuées d'une part sur les trois sources azotées testées, d'autre part sur le régime protéiprive. La déshydratation en cours de stockage des constituants du régime protéiprive, notamment de l'amidon de maîs, s'est traduite par une élévation de sa teneur en matière sèche entre le moment de sa fabrication $(88,9)$ et sa distribution $(90,3)$, de sorte qu'il a été nécessaire d'appliquer une correction. Il est possible par ailleurs, que les animaux, lors de la seconde phase de l'étude soient mieux adaptés aux conditions expérimentales, phénomène déjà observé par HENRY et DE WILDE (I973). Les valeurs énergétiques ainsi obtenues sont légèrement inférieures à celles rapportées par ailleurs (HENRY et BouRdon, I973) mais 1'écart entre les trois sources azotées est conservé : c'est ainsi que la valeur énergétique de la féverole entière est plus faible que celle du tourteau de soja 44 (différence de $300 \mathrm{kcal}$ $\mathrm{ED} / \mathrm{kg} \mathrm{MS}$ ), tandis que la valeur énergétique de la féverole décortiquée est comparable à celle du tourteau de soja 44 (4 040 contre $4000 \mathrm{kcal} \mathrm{ED/kg} \mathrm{MS).}$

\section{2. - Digestibilité des acides aminés du tourteau de soja, de la féverole entière et de la féverole décortiquée}

Les résultats portant sur la digestibilité apparente ou réelle des acides aminés ne mettent pas en évidence un acide aminé indispensable dont la digestibilité soit nettement plus faible que les autres. Ceci n'a pas été retrouvé dans l'étude de MEIER, POPPE et WrESEMÜLLER (r970), qui, avec un régime à base d'orge, ont observé une digestibilité plus faible de la lysine et de la tyrosine : respectivement 57 et 54 p. Ioo. Dans l'ensemble, les coefficients d'utilisation digestive apparents des acides aminés sont en accord avec les valeuts trouvées par Dammers (I964), NiELSEN (I968), EGGUM (I973). Dans ces différentes études, les acides aminés indispensables dont la digestibilité est la plus élevée sont l'arginine et l'histidine et à un moindre degré, la lysine. Inversement, la thréonine, la valine et l'isoleucine semblent toujours avoir une digestibilité plus faible que celle de l'azote. Dans le groupe des acides aminés non indispensables, l'acide glutamique présente souvent une digestibilité plus élevée, au contraire de l'alanine. Des réactions de transamination entre ces deux acides aminés, dans la lumière intestinale, peuvent expliquer ces différences qui sont d'ailleurs retrouvées quand on étudie la cinétique d'apparition des aminoacides dans la veine porte, après un repas (Pron et al., I964). Enfin, en ce qui concerne l'excrétion fécale endogène d'azote et d'acides aminés (tabl. 7), deux remarques peuvent être formulées. D'une part, la quantité d'azote endogène, exprimée en g pour Ioo g de matière sèche ingérée, semble légèrement inférieure aux valeurs obtenues par Dammers (I964) ou PoppE, Meier et Wiesemüllier (I970). D'autre part, la composition en acides aminés de l'azote endogène n'apparaît pas, en général, différente de celle trouvée par DAMMERs (I964) ou par CARL,SON et BAYLEY (I970).

\section{3. - Disponibilité des acides aminés, utilisation métabolique de l'azote}

L'examen des variations des concentrations en acides aminés libres du sang peut représenter un moyen d'étude de la disponibilité des acides aminés indispensables 
pour satisfaire le besoin de croissance de l'animal. Au moment où les prélèvements de sang systémique ont été effectués, la concentration en chaque acide aminé représente l'équilibre entre l'apport alimentaire et la soustraction du plasma qui est fonction du besoin de l'organisme en cet acide aminé (BoomgaARd et McDonaln, I969; TyPPo et al., I970 ; RÉRAT, I97I). Dans le cas présent, la quantité journalière d'azote retenu, qui est en relation étroite avec la vitesse de croissance des animaux (fig. I),

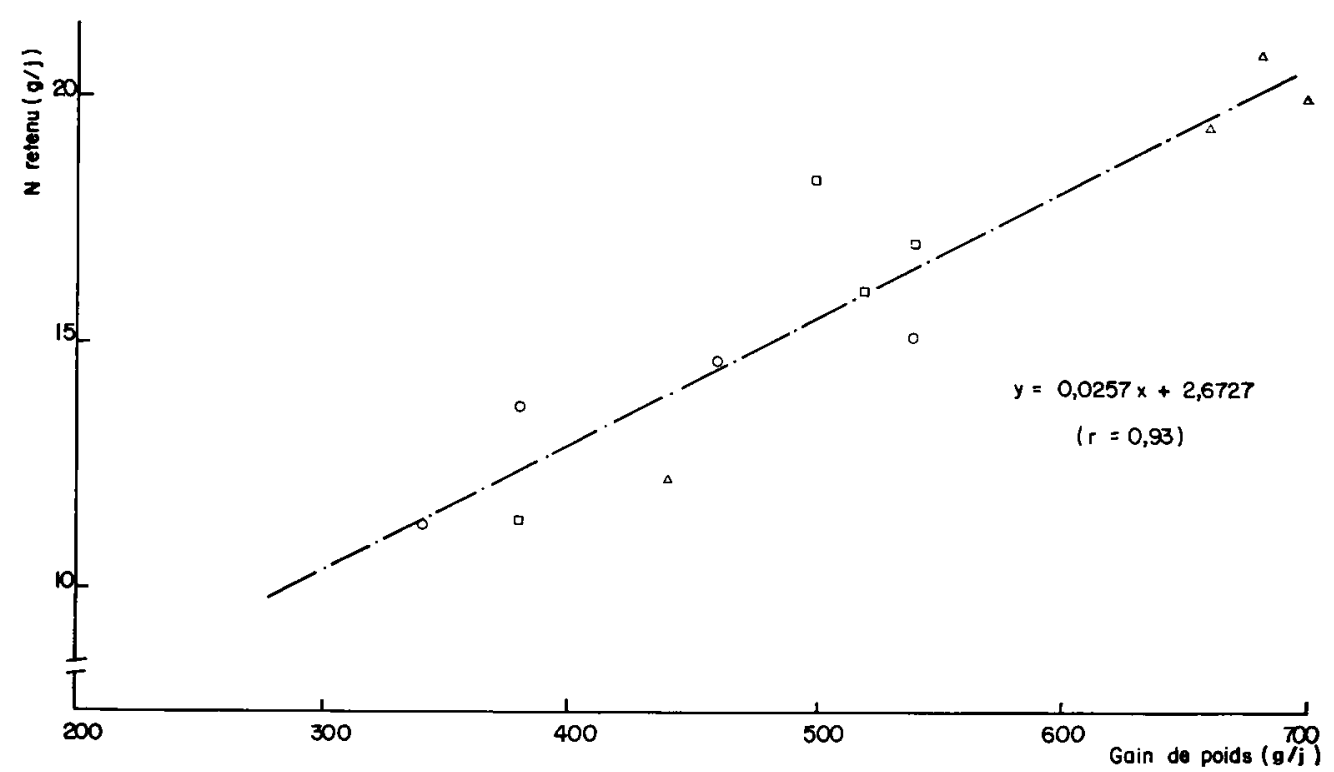

FIG. I. - Relation entre l'azote retenu et la vitesse de croissance des animaux

$\triangle-\triangle$ tourteau de soja (lot I)

$\square-\square$ féverole entière (lot 2)

- o féverole décortiquée (lot 3 )

semble plus faible dans le lot 3 (féverole décortiquée). Or, les résultats du tableau 8 mettent en évidence, dans ce lot, une augmentation de la somme des acides aminés indispensables libres dans le sang, tout particulièrement de la lysine. Il existe, d'ailleurs, une augmentation linéaire de la teneur en lysine libre, quand la quantité d'azote retenu diminue (fig. 2). D'autre part, les teneurs en méthionine et en thréonine libres du sang semblent plus faibles dans le lot I (tourteau de soja). Indépendamment d'une croissance plus élevée des animaux de ce lot qui explique, comme, ci-dessus, les taux sanguins plus faibles en certains acides aminés indispensables, deux points peuvent être soulignés. Le premier est que, pour la méthionine, les concentrations plus élevées dans les lots 2 et 3 (féverole entière et décortiquée) sont également la conséquence d'une teneur de la ration en méthionine sous forme libre plus importante (supplémentation des régimes 2 et 3 par $0, I_{5} \mathrm{p}$. roo de DL-méthionine).

Le second point est qu'une teneur plus faible en thréonine libre dans le sang des: animaux du lot I, malgré un taux plus élevé de cet acide aminé dans leur ration semblerait indiquer que la thréonine est le facteur limitant du tourteau de soja après supplémentation en méthionine. Inversement, les teneurs plus élevées en thréonine 
libre dans les lots 2 et 3 , outre qu'elles traduisent une vitesse de croissance et une quantité d'azote retenu plus faible, signifient qu'il pourrait exister un autre acide aminé limitant, par exemple le tryptophane. Cette carence probable en tryptophane dans les régimes $z$ et 3 pourrait d'ailleurs être plus marquée dans le cas de la féverole décortiquée, compte tenu d'un taux de matières azotées plus faible dans le régime et d'une diminution concomitante de la quantité d'azote ingéré. Ceci explique, vraisemblablement, la diminution de l'utilisation métabolique de l'azote, à 30 comme à $65 \mathrm{~kg}$, dans le lot 3 .

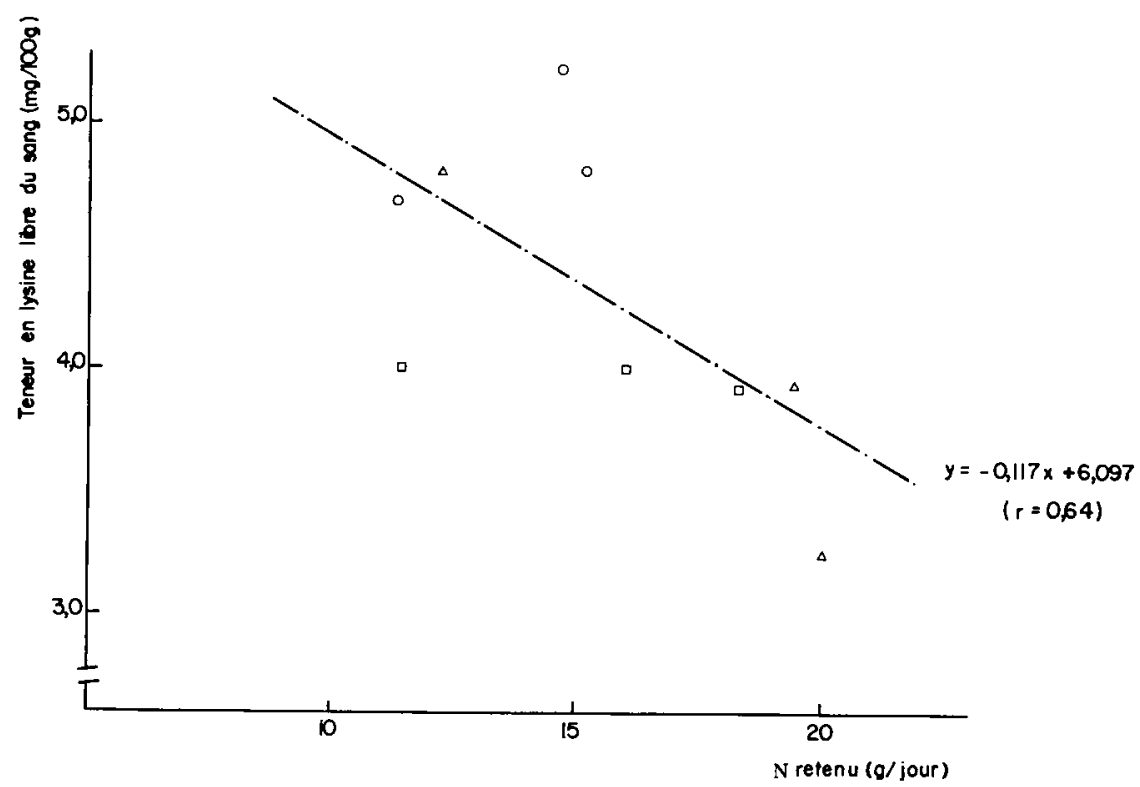

FIG. 2. - Relation entre la teneur en lysine libre du sang et l'azote retenu par l'animal

$\Delta-\Delta$ tourteau de soja (lot I)

$\square-\square$ féverole entière (lot 2)

○- o féverole décortiquée (lot 3 )

Sur le plan méthodologique, il apparait ainsi nécessaire d'associer aux variations de concentrations en acides aminés libres du sang, le niveau des performances des animaux et, en particulier, la quantité journalière d'azote retenu.

En conclusion, les résultats de cette étude démontrent que l'incorporation de féverole entière à un taux élevé dans la ration (5I p. IOo) dépréciel'utilisation digestive des nutriments (énergie, azote, acides aminés). Son remplacement total par la féverole décortiquée améliore cette utilisation digestive, qui devient comparable à celle obtenue avec un régime renfermant $d u$ tourteau de soja, mais cet effet bénéfique ne s'observe plus au niveau de l'utilisation métabolique de l'azote. Quoi qu'il en soit cette dernière observation doit être confirmée sur un effectif plus important d'animaux. 


\section{SUMMARY}

\section{UTILIZATION OF WHOLE AND SHELLED HORSE-BEAN BY THE GROWING PIG : DIGESTIBILITY AND AMINO ACID AVAILABILITY}

The present study was made with $\mathrm{r} 2$ castrated male pigs of the Large White breed, weighing on an average $25 \mathrm{~kg}$ and kept in metabolism crates in order to compare the apparent digestibility and metabolic utilization of horse-bean of the variety Pavane, either whole (group 2) or shelled (group 3) with those of soybean oil meal 44 (group I). These feeds constituted the only protein source and were incorporated into semi-purified diets in replacement of variable proportions of maize starch with the aim of obtaining identical crude protein contents. In addition, the diets were supplemented with DL-methionine at a rate of 0.05 and $0.5_{5}$ p. Ioo, respectively for " soybean " and " horse-bean " diets.

The apparent digestibility of the dietary components was lower in group 2 and, as compared with group $\mathrm{I}$, this lowering was larger for protein $(78.80$ versus 89.49$)$ than for energy (84.49 versus 89.79).

Shelling of the horse-bean seeds improved the digestibility of the various nutrients (protein : 86.r 7 ; energy : 89.79). On the other hand, the metabolic utilization of the nitrogen absorbed was lower in group 3 (retention coefficient : $5 \mathrm{I} .26 \mathrm{p}$. roo) than in the two other groups $(64.73$ in group I ; 60.04 in group 2). As a matter of fact, the daily nitrogen retention, closely related to the growth rate of the animals, was higher in group I ( $18.10 \mathrm{~g}$ ) than in the two horse-bean groups (group 2 : I 5.69 ; group $3:$ I 3.68 ).

All these results were confirmed at a later stage of growth $(65 \mathrm{~kg})$.

The apparent and real digestibilities of the amino acids from the three protein sources were estimated and it appeared that the magnitude of their variations was higher in group 2 (whole horse-bean). In the 3 groups, the apparent or real digestibility of arginine, histidine, and glutamic acid was higher than that of nitrogen ; conversely, threonine, valine, isoleucine, alanine and glycine exhibited a lower apparent or real digestibility.

Finally, measurement of the blood levels of free amino acids showed a higher free lysine content in group 3 in relation with a lower nitrogen retention.

\section{RÉFÉRENCES BIBLIOGRAPHIQUES}

Boomgaardt J., McDonald B. E., I969. Comparison of fasting plasma aminoacid patterns in the pig, rat and chicken. Can. J. Physiol. Pharmacol., 47, 392-395.

CARLSON K. H., BAYLeY H. S., I97o. Nitrogen and amino acids in the feces of young pigs receiving a protein-free diet and diets containing graded levels of soyabean oil meal or casein. J. Nutr., 100, I353-I36r.

Dammers J., r964. Étude de la digestibilité chez le Porc. Les facteurs qui infuencent la digestibilitê des aliments. Digestibilité des aminoacides. Thèse DrUKKERI "West Friesland "Hoorn, Universiteit Van Leuven, Landbouvinstituut.

Duéz P. H., Rérat A., 1975. Étude du besoin en lysine de la Truie gestante nullipare. Ann. Zootech. (sous presse).

EGGUM B. O., I973. A study of certain factors influencing protein utilization in rats and pigs. 406 Beretning fra forogslaboratoriet Udgivet af Statens Husdyrbrugsudvalg. Kobenhavn.

Henry Y., Bourdon D., 1972. Essai de remplacement du tourteau de soja par la féverole dans l'alimentation du porc en croissance-finition. Journées Rech. porcine en France, Paris éd. I. T. P., I75-183.

Henry Y., Bourdon D., 1973. Utilisation digestive de l'énergie et des matières azotées de Ia féverole sous forme entière ou décortiquée, en comparaison avec le tourteau de soja. Journées Rech. porcine en France, Paris éd. by I. T. P., I05-II4.

HeNRY Y., DE WILDE R., r973. Incorporation de proportions variables de matières grasses (huile d'arachide) dans le régime du porc en croissance-finition, en relation avec le taux de matières azotées. I. Influence sur l'utilisation digestive des constituants énergétiques et azotés, et la rétention azotée. Ann. Zootech., 22, 167-184.

Henry Y., Rérat A., Ig66. Utilisation des pommes de terre deshydratées et fraîches dans l'alimentation du porc en croissance en comparaison avec l'orge. Ann. Zootech., 15, 23r-25I. 
Meier H., Poppe S., Wiesemüller W., I970. Untersuchungen über die Aminosaürenresorption aus verschiedenen Proteinträgern beim Schwein. II. Pflanzliche Futterstoffe. Arch. Tierernährung, 20, $567-574$.

Nielsen A. J., I968. Digestibility of Amino Acids in relation to digestibility of Nitrogen in bacon pigs. Royal veterinary and Agricultural College. Copenhagen, Denmark. Yearbook, 79-88.

Pion R., Fauconneau G., I966. Les acides aminés des protéines alimentaires. Méthodes de dosage et résultats obtenus. Cahier no 6 Amino acides, peptides, protéines, 157-175, A. E. C. Commentry.

Pion R., Fauconneau G., Rérat A., rg64. Variation de la composition en acides aminés du sang porte au cours de la digestion chez le Porc. Ann. Biol. anim. Bioch., Biophys., 4, 383-4or.

Poppe S., Meier H., Wiesemüller W., I97o. Untersuchungen über die Aminosaürenresorption aus Verschiedenen Proteinträgern beim Schwein. III. Bestimmung der Darmverlustaminosaüren. Arch. Tiereynährung, 20, 575-582.

Rérat A., I97I. La valeur biologique des protéines : quelques acquisitions récentes. Ann. Zootech., 20, $193-246$.

Schimmann R., Hofrmann L., Nehring K., ig6r. Die Verwertung reiner Nährstoffe. II. Versuche mit Schweinen. Arch. Tierernährung, 11, 265-283.

Toullec R., Flanzy J., Rigaud J., I968. Dosage des lipides des fèces. Ann. Biol. anim. Bioch., Biophys., 8, 28I-289.

Typpo J. T., Meade R. J., Nordstrom J. W., Stockland W. L., r97o. Influence of time of fast on concentrations of free amino acids in plasma and total amino acids in liver protein of young swine. J. Anim. Sci., 31, 885-893.

VERMOREL M., I973a. Utilisation énergétique et azotée des principaux tourteaux et des graines de légumineuses par le Rat en croissance; comparaison au blé tendre et au blé dur. Ann. Zootech., 22, 253-265.

Vermorel M., i973 b. Influence du décorticage sur la valeur nutritive de la féverole. Premiers résultats obtenus chez le Porc et le Rat en croissance. Bulletin technique du C. R. V. Z., S. E. I. éd., I5-I8.

ZEBrowska T., r973. Influence of dietary protein source on the rate of digestion in the small intestine of pigs. II. The rate of protein digestion and amino acid absorption. Roczniki Nauk Roln., B-95-I, I35-I 55 . 